\title{
Full-Thickness Abdominal Skin Graft for Long-Segment Urethral Stricture Reconstruction
}

\author{
Joshua J. Meeks, Bradley A. Erickson, Chris M. Gonzalez \\ Department of Urology, Northwestern University, Feinberg School of Medicine, Chicago, Illinois, \\ USA
}

\begin{abstract}
Multiple tissue sources have been used for urethral reconstruction in adults. Patients with lichen sclerosis (LS), long segment strictures, or prior oral graft use have less available tissue for urethroplasty. We describe a technique for the use of a full-thickness skin graft of hairless abdominal skin for long segment urethroplasty.
\end{abstract}

Key words: skin grafting, urethroplasty

Int Braz J Urol. 2008; 34: 602-8

\section{INTRODUCTION}

Reconstruction of long-segment adult urethral stricture disease as a result of lichen sclerosis (LS) or failed pediatric hypospadias repair remains a difficult urological problem mainly due to the deficiency of available extra-genital skin for grafting (1). Graft tissue has been utilized successfully for urethral reconstruction from various sites including buccal mucosa, genital skin and auricular tissue; however each tissue source has specific drawbacks (2). The ideal graft source would be extra-genital in origin, hairless, produce minimal postoperative morbidity at the harvest site, be inconspicuous postoperatively and abundant enough in length and width so as to avoid multiple urethral suture lines for men with long segment strictures. In an effort to develop an alternative to available graft sources for complex and long-segment strictures we describe harvest and application of a full-thickness abdominal skin graft for urethral reconstruction.

\section{SURGICAL TECHNIQUE}

The extent of the urethral stricture was evaluated preoperatively with cystoscopy and retrograde urethrogram. The area of abdominal wall to be harvested was demarcated and discussed with the patient preoperatively. Deep venous thrombosis precautions were taken, and all patients achieved a sterile urine culture prior to surgery.

For one or two-staged long segment urethral reconstructive procedures involving the mid or proximal bulbar urethra, the patient was placed in the low lithotomy position. Otherwise, those with stricture confined to the penile urethra and distal bulbar urethra were placed in the supine position. A ventral longitudinal shaft incision is made to expose the penile urethra to the level of the scrotum in men undergoing single-staged repair with a perineal counter-incision to access the bulbar urethra if needed. A bougie-à-boule sound is then used to identify the anatomically distal most aspect of the stricture. The 
urethra is incised along its anterolateral edge throughout the length of the stricture with the edges of the urethrotomy calibrated to $24 \mathrm{~F}$ in the penile urethra and $26 \mathrm{~F}$ for the bulbar urethra. The full extent of the urethrotomy is then measured in preparation for graft harvest. Alternatively, in men undergoing the first of a two-staged procedure for long segment stricture disease, a grooved director is placed within the urethra and a scalpel is used to open the urethra through the full thickness of the penile skin and urethra. The mucosa from the proximal urethrotomy site is then sutured to the overlying penile, scrotal, or perineal skin, depending on stricture length, with interrupted 5-0 vicryl sutures. All nonviable corpus spongiosum and urethral mucosa or tissue that is suspicious for lichen sclerosis is excised and sent for pathologic analysis in both single and two staged procedures.

Graft harvest of the abdominal wall involves excision of the skin of the right or left lower quadrant of the abdomen at the level of the anterior superior iliac crest (Figure-1). An area of hairless skin is identified and chosen in a location which is anatomically
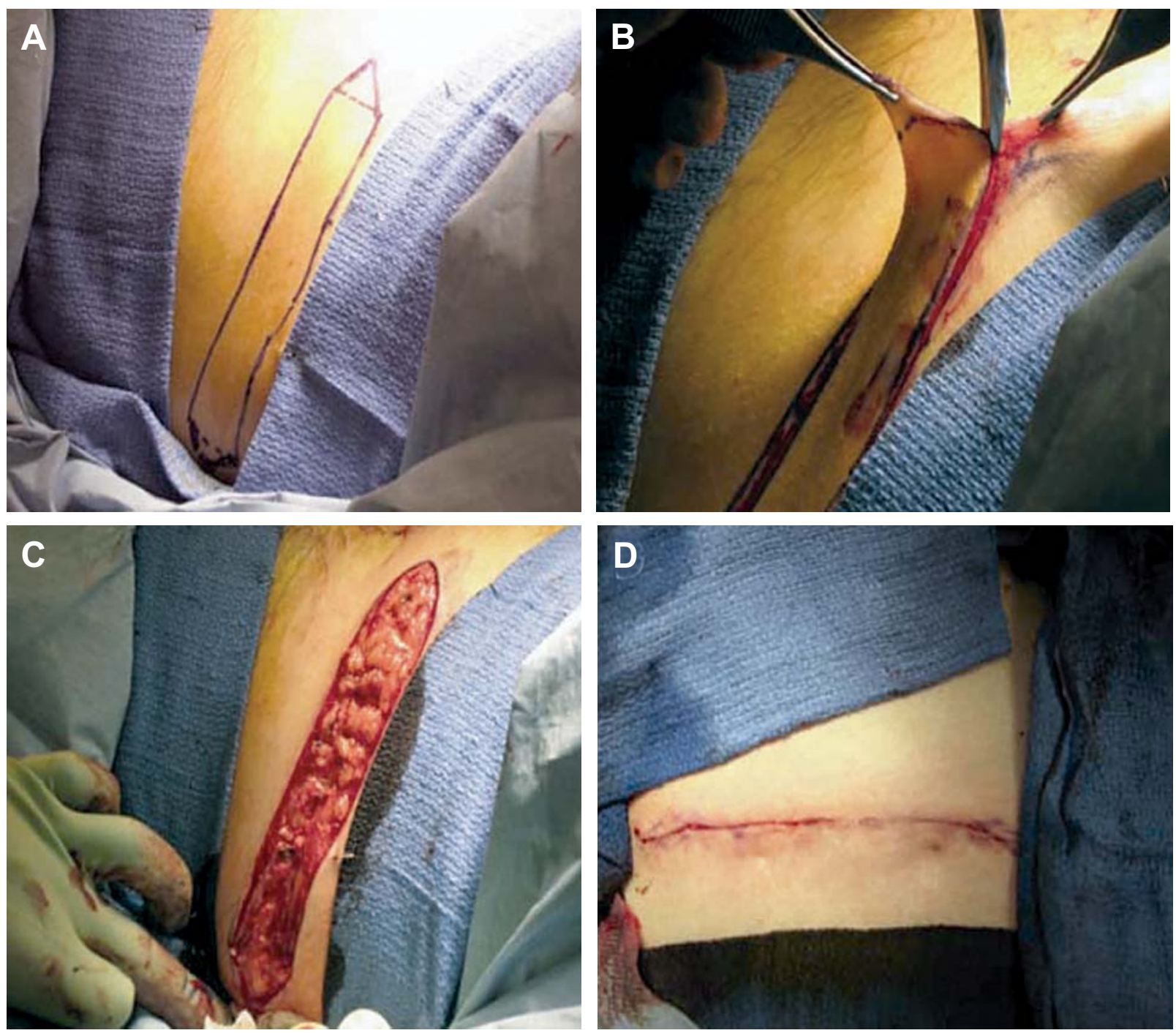

Figure 1 - Harvest of a full-thickness abdominal skin. A)- Region of the abdominal skin is marked that is hairless on a region and is covered by the patient's belt. B) and C)-A full thickness skin graft is harvested. D)- The graft site is closed with deep dermal and subcutiticular sutures. 
positioned so that the patient's belt line will eventually conceal the wound (Figure-1A). A full thickness skin graft is harvested to the level of the subcutaneous tissue (Figure-1B and Figure-1C). Once the graft is sharply excised, the deep dermal tissue of the harvest site is closed with interrupted 3-0 vicryl sutures followed by a 4-0 vicryl subcuticular skin closure (Figure-1D). The graft is then prepared by sharp dissection over its dermal edge until transparent (Figure-2A).

For single-stage procedures, the epithelial side of the graft is sutured to the remaining mucosa of the urethral plate with 5-0 vicryl suture. In the first of a two-staged procedure, graft fixation to the dartos fascia is accomplished by suturing the graft to the urethral plate medially and the penile skin laterally (Figure-2B). Venting incisions or "pie crusting" is then completed through the graft, and quilting sutures are placed per square centimeter in order to fix the graft to the underlying dartos and corpora cavernosum to enhance inosculation and prevent sub-graft fluid collections (Figure-2B).

A $16 \mathrm{~F}$ catheter is left in place for strictures limited to the penile urethra and an $18 \mathrm{~F}$ catheter is placed for strictures extending into the bulbar urethra. All men undergoing the first of a two-staged procedure had a catheter placed for 5 days postoperatively in conjunction with a moisturized bolster dressing. All other patients undergoing a single procedure for repair had catheter drainage for three weeks postoperatively.

\section{RESULTS}

Abdominal skin was used in ten patients with long-segment urethral stricture disease (Table-
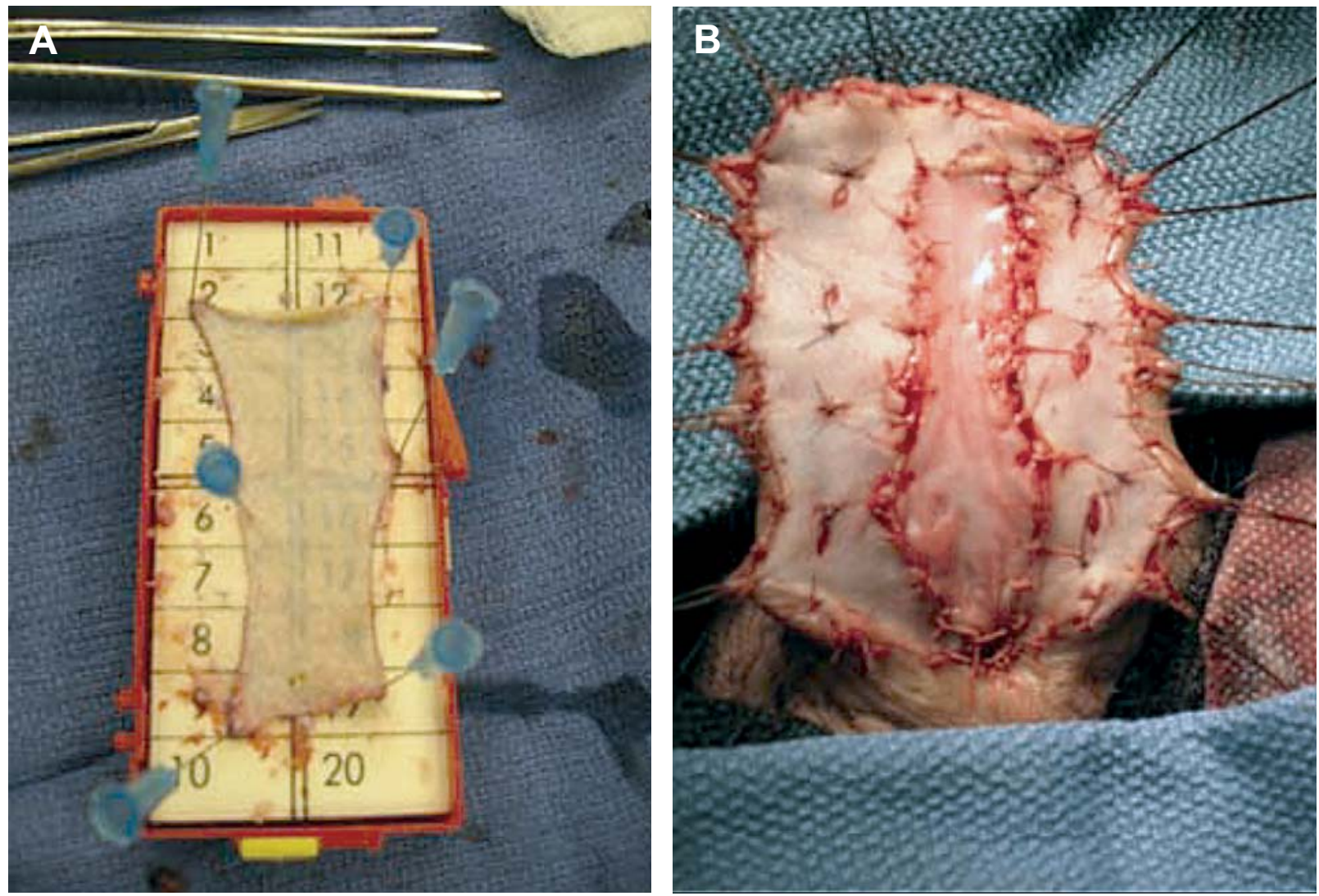

Figure 2 - Processing and placement of full-thickness abdominal skin graft. A)- The harvested abdominal wall graft is thinned to transparency. B)- The graft is sewn into place around the urethral plate. The graft is then pie crusted and quilting sutures are placed. 
Abdominal Wall Skin Graft

Table 1 - Clinical demographics of patients undergoing abdominal wall skin graft.

\begin{tabular}{|c|c|c|c|c|c|c|c|c|c|}
\hline Patient & Age & Cause & $\begin{array}{c}\text { Length } \\
(\mathrm{cm})\end{array}$ & $\begin{array}{l}\text { Graft Size } \\
\quad(\mathrm{cm})\end{array}$ & $\begin{array}{l}\text { Graft } \\
\text { Area } \\
\left(\mathrm{cm}^{2}\right)\end{array}$ & $\begin{array}{l}\mathbf{E B L} \\
\text { (cc) }\end{array}$ & $\begin{array}{l}\text { Type } \\
\text { Repair }\end{array}$ & Recurrence & Comp \\
\hline 1 & 18 & $\begin{array}{l}\text { penoscrotal } \\
\text { hypospadias }\end{array}$ & 10 & $10 \times 3$ & 30 & 350 & 2-stage & & \\
\hline 2 & 48 & $\begin{array}{l}\text { penoscrotal } \\
\text { hypospadias }\end{array}$ & 11 & $11 \times 3.5$ & 38.5 & 100 & 2-stage & & UTI \\
\hline 3 & 54 & LS & 12 & $14 \times 4$ & 56 & 400 & 2-stage & & \\
\hline 4 & 32 & LS & 8 & $8 \times 3$ & 24 & 300 & 2-stage & & hair \\
\hline 5 & 77 & LS & 24 & $15 \times 4$ & 60 & 600 & 1-stage & $\begin{array}{l}11 \text { months; } \\
2 \mathrm{~cm} \\
\text { proximal } \\
\text { bulbar } \\
\text { urethra }\end{array}$ & \\
\hline 6 & & LS & 22 & $20 X 4$ & 80 & 800 & 1-stage & & hair \\
\hline 7 & 55 & unknown & 19 & $19 \times 2$ & 38 & 450 & & & \\
\hline 8 & 47 & LS & 10 & $10 \times 4$ & 40 & 750 & 2-stage & & UTI \\
\hline 9 & 45 & unknown & 12 & $12 \times 2$ & 24 & 200 & 1-stage & & \\
\hline 10 & 55 & LS & 21 & $14 \times 3$ & 32 & 600 & 1-stage & $\begin{array}{c}6 \text { months; } \\
2 \mathrm{~cm} \text { distal } \\
\text { bulbar } \\
\text { urethra }\end{array}$ & \\
\hline
\end{tabular}

Comp. $=$ complications; $E B L=$ estimated blood loss; $L S=$ lichen sclerosis; $U T I=$ urinary tract infection.

1). Median patient age was 42 years (range 18-77 years). Mean stricture length was $12 \mathrm{~cm}$ (range 10$24 \mathrm{~cm})$. The etiology of urethral stricture included failed hypospadias repair (2), LS (6) and unknown (2). Median follow-up was 17 months (range 3-25 months). Average graft area was $42.25 \mathrm{~cm}^{2}$. Six of the ten patients underwent two-staged procedures with successful graft uptake in all men after the first stage and successful second stage closure in the two men completing both procedures (Figure-3A and Figure-3B). In the other four men, strictures were closed in one stage with a long segment graft. Mean estimated blood loss was $412 \mathrm{cc}$. Two patients with LS developed recurrent stricture formation at a mean time of 9 months from surgery. These were the first two patients in this series with prior urethroplasty utilizing buccal and auricular tissue and involved stricture lengths of 21 and $24 \mathrm{~cm}$. Recurrent stricture length was $2 \mathrm{~cm}$ in each patient and was managed endoscopically.

All abdominal skin harvest sites healed well without complication. Two patients developed febrile urinary tract infections requiring oral antibiotics. Two patients grew hair from segments of the abdominal skin graft within six months of surgery, one after single stage urethroplasty and the other after the first of a two-staged procedure. These were the first two grafts harvested in this series when areas with hair were taken and the follicles removed. This method proved to be unsuccessful in its ability to prevent all future hair growth, and all subsequent grafts were harvested from hairless abdominal regions with no further occurrences of hair growth on the graft. All patients in this series were discharged on postoperative day one and reported minimal pain at the abdominal harvest site. 

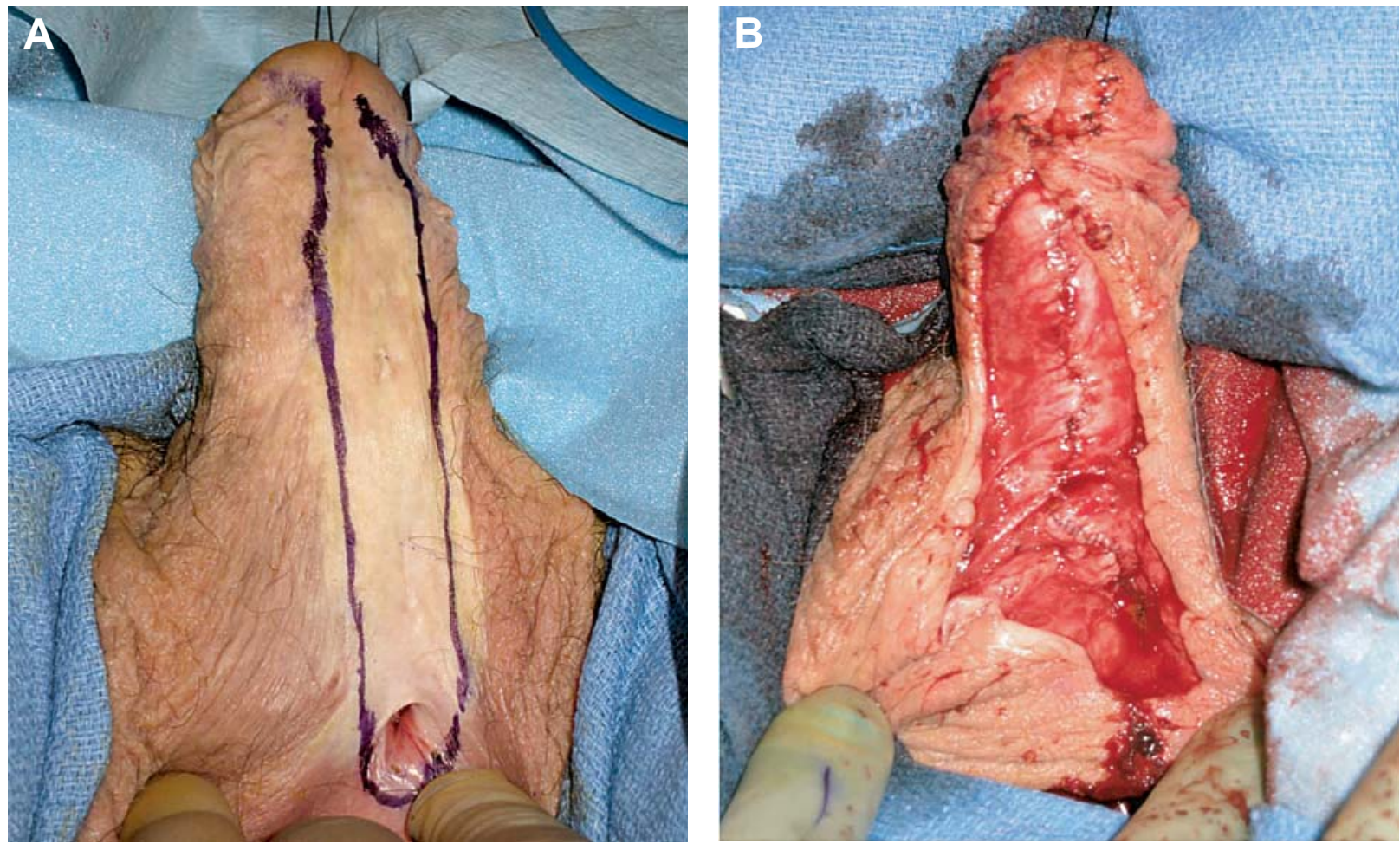

Figure 3 - Second state urethroplasty. A) full-thickness abdominal skin graft six months after first stage urethroplasty, prior to second stage urethroplasty. B)- Rolled urethral tube after second stage urethroplasty.

\section{COMMENTS}

Urethral reconstruction in patients with long segment stricture disease remains a complicated surgical problem especially in men with previous hypospadias surgery and those with LS as an etiology. The success rates of reconstruction in men with longer segment strictures of varied etiology has been reported to be $75 \%$ at five years with a median stricture length of $7 \mathrm{~cm} \mathrm{(3).} \mathrm{Urethral} \mathrm{reconstruction} \mathrm{for} \mathrm{long} \mathrm{segment}$ stricture disease after previous hypospadias repair has a similar $75 \%$ success rate at nearly three years (4). Men with long-segment stricture disease secondary to LS appear to have a higher recurrence rate secondary to the progressive nature of this inflammatory disorder.

One of the critical events for urethral reconstruction of long-segment strictures involves obtaining the appropriate tissue for urethral defect substitution. Harvest of tissue from the surrounding penile skin is ideal, but this tissue is often deficient, scarred, or may be at risk for recurrence of LS. To repair long-segment defects, some authors have used composite repairs including genital fasciocutaneous flaps in conjunction with buccal or penile skin grafts. Berglund and Angermeir described the use of a combined penile or scrotal skin flap with buccal mucosa grafts in patients with strictures up to $24 \mathrm{~cm}$ in length with a success rate of $83 \%$ approaching 6 years of follow-up (5). While these authors were able to obtain good results from these techniques for long-segment strictures some of the potential drawbacks include the need to harvest graft tissue from multiple sites, the risk of suture line ischemia secondary to incorporation of multiple grafts into the anastomosis, the risk of hair growth on genital graft or flap tissue, and utilization of genital skin which may predispose to an LS related stricture recurrence.

Buccal mucosa alone has been demonstrated to be a good choice for extra-genital graft tissue with success rates reaching $90 \%$ in some series. However, a significant limitation of buccal mucosa for substitution in long segment stricture reconstruction involves the availability of this tissue. The cumulative length of 
available oral mucosa is approximately $17 \mathrm{~cm}$ which requires harvest from both cheeks and potentially the lower lip. Complications reported with oral harvest from just one site include neurosensory deficits, changes of salivary flow, difficulty with mouth opening, and lip contracture $(5,6)$. In one report, as many as $26 \%$ of men indicated negative or mixed feelings about the buccal mucosa graft harvest postoperatively with $16 \%$ reporting persistent numbness and $32 \%$ reporting oral tightness (2). Comparatively, we experienced no harvest site morbidity and limited patient complaints related to the abdominal wall harvest for long-segment strictures with a mean length of $12 \mathrm{~cm}$.

The use of non-oral, extra-genital FTSG has previously been described in several smaller series for urethral reconstruction with reported success ranging from $25-50 \%$ (7). These outcomes may have been related, in part, to the widespread use of tubegrafts for reconstruction at this time, and the choice of single versus two-staged repair for complicated strictures. The use of full-thickness abdominal wall skin for long-segment stricture reconstruction has not been described previously. The advantages of this tissue are that it provides extra-genital tissue origin, the ability to harvest hairless segments up to $24 \mathrm{~cm}$, and the limited graft site morbidity observed in this series. Furthermore, the abundant length and width of the abdominal skin graft allows for harvest of a single graft segment as compared to buccal mucosa or penile skin grafts which require multiple harvest sites and suture lines between grafts within an anastomosis for long-segment stricture defects. While abdominal skin tissue should not be the first choice for graft tissue until long-term outcomes are known, the availability of this tissue offers the reconstructive surgeon an additional option for substitution urethroplasty of long-segment strictures.

\section{CONCLUSION}

We describe a technique for full-thickness abdominal skin graft use in long-segment urethral stricture reconstruction. When harvested from hairless regions, these grafts have acceptable success rates with few complications at early follow-up. Selective use of abdominal skin grafts may be well suited for patients with long-segment urethral strictures in one or two stages when other graft sources are not available or feasible.

\section{CONFLICT OF INTEREST}

None declared.

\section{REFERENCES}

1. Xu YM, Qiao Y, Sa YL, Wu DL, Zhang XR, Zhang J, et al.: Substitution urethroplasty of complex and longsegment urethral strictures: a rationale for procedure selection. Eur Urol. 2007; 51: 1093-8; discussion 1098-9.

2. Dublin N, Stewart LH: Oral complications after buccal mucosal graft harvest for urethroplasty. BJU Int. 2004; 94: 867-9.

3. Moradi MR, Moradi A: Urethroplasty for Long Anterior Urethral Strictures: Report of Long-term Results. Urol J. 2006; 3: 160-4.

4. Barbagli G, De Angelis M, Palminteri E, Lazzeri M: Failed hypospadias repair presenting in adults. Eur Urol. 2006; 49: 887-94; discussion 895.

5. Berglund RK, Angermeier KW: Combined buccal mucosa graft and genital skin flap for reconstruction of extensive anterior urethral strictures. Urology. 2006; 68: 707-10; discussion 710.

6. Jang TL, Erickson B, Medendorp A, Gonzalez CM: Comparison of donor site intraoral morbidity after mucosal graft harvesting for urethral reconstruction. Urology. 2005; 66: 716-20.

7. Webster GD, Brown MW, Koefoot RB Jr, Sihelnick S: Suboptimal results in full thickness skin graft urethroplasty using an extrapenile skin donor site. J Urol. 1984; 131: 1082-3.

$\overline{\text { Accepted after revision: }}$ June 4, 2008 


\section{EDITORIAL COMMENT}

The authors reported on the use of full-thickness abdominal skin graft for urethral strictures as an "inlay" after extensive urethrotomy for long strictures. The paper was submitted as an operative technique description focusing mainly in the procedure itself with a limited number of patients treated (10) and only 6 completing the second-stage. Follow-up is also very short (17 months).

The paper has some merit but adds little to the present knowledge in urethroplasty. Since the popularization of mucosal grafts in urethral structure and hypospadias repair, there is a consensus that mucosal grafts are more appropriate and recently tunica vaginalis is also being studied as a valid option. Skin grafts have been extensively studied in the past with success and reported in the literature also with long term follow-up. Bracka has shown long-term clinical data in over 1000 patients with skin grafts and later buccal mucosa grafts including the second-stage urethroplasty. The argument of the authors that the suggested donor area is attractive should be based on clinical results in a larger series and not only on surgical technique descriptions.

In summary, I would like to encourage the authors to resubmit their experience later with more patients and a longer follow-up.

Dr. Antonio Macedo Jr. Federal University of Sao Paulo Sao Paulo, SP, Brazil E-mail:amcdjr@uol.com.br

\section{REPLY BY THE AUTHORS}

In this surgical technique manuscript, we describe the procedure to harvest, prepare and place a full-thickness skin graft for men with long segment urethral strictures. While long segment urethral reconstruction is relatively rare, the most difficult part of urethroplasty is finding an ideal tissue source for urethral reconstruction. Many have described the use of genital skin, non-genital skin and buccal mucosa as graft sources; these standard tissues sources are often deficient in men with prior urethroplasty or pediatric hypospadias repair, as several men in our study were. In our study, the mean stricture length was $12 \mathrm{~cm}$ with a range of 10 to $24 \mathrm{~cm}$. In this population, the potential graft sources include composite grafts of multiple buccal grafts with the possible addition of skin grafts. Yet, almost $25 \%$ of men describe a complication after buccal harvest. The technique we describe is not meant to replace standard techniques of buccal or genital skin grafts, but is a supplemental technique to consider when approaching a complicated patient with few ideal graft sources. As the editor mentions, more data will be forthcoming.

The Authors 